\title{
Il Diritto, La Scienza e La TeCnologia ${ }^{1}$
}

\author{
Stefano Magnolo* \\ Alessandro Taurino** \\ 1 Introduzione. 2 Evoluzione. 3 Tecnologia. 4 Intelligenza artificiale. 5 Conclusioni. \\ Referências.
}

\section{RIASUNTO}

Le nuove tecnologie dell'informazione e della comunicazione giocano un ruolo rilevante per i sistemi sociali coinvolti. Per questo la descrizione teorica della società non può trascurare la dipendenza sempre maggiore della società moderna dalla tecnologia. Il nostro articolo si prefigge l'obiettivo di esaminare il progresso tecnologico dal punto di vista della evoluzione degli strumenti giuridici regolativi delle nuove tecnologie. Si tratta di un punto di vista sociologico-giuridico dove è in gioco la dinamica dell'evoluzione del diritto rispetto alla evoluzione della società. Diversamente da altri settori del diritto, esempio classico il diritto di famiglia, qui non c'è una tradizione consolidata alla quale riferirsi o da rigettare. Ciò significa che le soluzioni devono essere "inventate" alla luce di paradigmi nuovi che, pur facendo appello a figure giuridiche fondamentali, abbiano una capacità visionaria, siano cioè, come dice Luhmann, "gravidi di futuro". Il nostro discorso partirà dunque da un inquadramento teorico generale delle dinamiche diritto-tecnologia-società per concludere avendo come riferimento il caso specifico della intelligenza artificiale.

Parole chiave: Progresso tecnologico. Evoluzione sociale. Law and Society. Human Enhancement Technologies. Intelligenza artificiale.

\section{INTRODUZIONE}

Questo articolo ha come oggetto l'osservazione delle dinamiche temporali nella dimensione sociale. Con ciò intendiamo riferirci alla questione delle trasformazioni strutturali che interessano la società nel suo complesso, alcuni ambiti della sua articolazione e le relazioni che intercorrono tra questi differenti livelli. Ma soprattutto intendiamo riferirci al fatto che ci poniamo al livello della osservazione di secondo ordine e infatti affermare che l'oggetto è costituito dalla osservazione di quelle dinamiche significa che noi osserviamo osservazioni

* Assistant Professor of Sociology of Law and Social Change, Facoltà di Lettere e Filosofia, Lingue e Beni Culturali, Università del Salento, Lecce - Italia. E-mail: <stefano.magnolo@unisalento.it〉. http://orcid.org/00000001-7252-8822

** Laurea in Giurisprudenza e diploma di Specializzazione in professioni legali presso l'Università del Salento, Lecce - Italia. Avvocato presso il foro di Lecce - Italia. https://orcid.org/0000-0001-5981-9289 


\section{(LUHMANN, 1995a; ESPOSITO, 1993).}

Da una parte avremo dunque a che fare con la dinamica delle relazioni diritto-società, dinamica che costituisce il riferimento oggettuale primario della sociologia del diritto. Dall'altra, dobbiamo distinguere due differenti livelli di interazione. Il primo concerne l'interazione che intercorre tra le innovazioni prodotte da un particolare sistema sociale, quale il sistema scientifico, e la "risposta" del sistema giuridico a tali innovazioni. Questa la rappresentazione della dinamica diritto-società nel caso particolare della scienza: il diritto moderno si adatta, si dice, alla mutata realtà sociale. Il secondo livello di interazione corrisponde alla dinamica delle trasformazioni innescate dallo sviluppo tecnologico, che ha un effetto particolare sull'ambiente umano e sulla comunicazione.

Il diritto moderno, come la semantica, segue necessariamente a distanza l'evoluzione della società. È fatto apposta, potremmo dire. Ciò che lo rende moderno è proprio la sua continua mutabilità, a differenza del diritto antico, legittimato dalla tradizione o del diritto naturale, universalmente valido ed eterno come l'istanza della sua legittimazione. La società cambia e il diritto moderno si adatta a questi cambiamenti. Esempio classico quello delle relazioni intime, che forse sono quelle che più immediatamente riflettono il cambiamento del modo di vivere e sentire della società. In questi casi il diritto riconosce, per esempio, altre forme di convivenza diverse dal matrimonio che si sono diffuse nella realtà sociale contemporanea. Certo, in misura e con modalità differenti nei vari paesi, a ulteriore conferma dell'assenza di modelli unici di riferimento e della sostanziale contingenza di tutte le norme, con una sola eccezione: le norme costituzionali. Le costituzioni, infatti, costituiscono un elemento divergente rispetto alla dinamica temporale del diritto ordinario. In qualche modo sono una sorta di "residuo" dell'antico diritto naturale, come dimostrano le difficoltà incontrate da Kelsen nella ricerca della loro fonte di legittimazione. ${ }^{2}$ La costituzione, infatti, non rispetta uno dei principi basilari delle codificazioni moderne, quello in base al quale il diritto nuovo è abrogativo di quello vecchio (TARELLO, 1976, p. 15-42). In questo senso, mentre per la scienza giuridica diventa problematica la legittimazione di un diritto ordinario temporalmente instabile, potremmo dire, la validità delle sue norme fino a che non ne intervengano di nuove, nel caso della costituzione il problema teorico diventa quello di un diritto che resta stabilmente valido nel futuro nonostante provenga, per così dire, dal passato. ${ }^{3}$

Ancora alcune ulteriori specificazioni prima di descrivere il punto di vista a partire dal quale ci muoviamo. La prima riguarda la scienza. Parliamo di sistema scientifico ad indicare sia le scienze hard, che nel nostro caso potrebbero essere la cibernetica, le biotecnologie, e le ICT, sia le scienze soft, quali le scienze umane e sociali. ${ }^{4}$ La nostra discussione si svolge al livello della scienza giuridica, della osservazione sociologica del diritto, nel caso specifico delle dinamiche diritto-società in chiave evolutiva, come chiariremo più avanti. La scienza entra quindi due volte nel nostro discorso. Questa precisazione serve a introdurre la seconda precisazione. È doveroso infatti chiarire come il nostro discorso è possibile solo a livello della scienza, di una scienza sociologica del diritto nel nostro caso, dato che intendiamo 
come separati l'ambito del diritto e l'ambito della politica, che è quello interessato dalla produzione normativa. Contrariamente ad una lunga tradizione che, sulla base del concetto politico di stato di diritto e della costituzione considerata come documento giuridico-politico, vede un sistema giuridico-politico unitario, noi intendiamo questi due ambiti come separati (LUHMANN, 1997, p. 407-439). È la proposta teorica di Luhmann, che rinviene proprio nell'esistenza della costituzione come documento giuridico-politico che contiene le norme (giuridiche) che regolano la produzione (politica) del diritto il meccanismo della separazione dei due ambiti e del loro modus operandi (LUHMANN, 1996). La logica che presiede a questa visione muove dalla constatazione che la distinzione dei due ambiti, quello politico e quello giuridico, è plausibile perché la loro differenziazione, basata sulla positivizzazione del diritto e la democratizzazione del potere politico conseguiti con le codificazioni moderne, non significa separazione o allontanamento, ma al contrario rende disponibili, grazie alla costituzione, possibilità di relazione tra i due ambiti così distinti (LUHMANN, 2002).

Se facciamo un passo indietro, vediamo come la nostra discussione solo apparentemente dovrebbe svolgersi a livello del sistema politico, in quanto investito appunto della selezione del materiale normativo astrattamente possibile. Tuttavia, come vedremo meglio più avanti, il discorso politico in quanto tale si svolge secondo logiche che qui non prenderemo in considerazione e che caratterizzano il momento della produzione del diritto. Questo momento si caratterizza come un procedimento, certo giuridicamente regolato abbiamo detto, che differisce sostanzialmente dai procedimenti giuridici e dalla loro logica e dove la discussione può svolgersi ammettendo le argomentazioni più disparate, ma che solo si conclude con il consenso della maggioranza sulla proposta selezionata (LUHMANN, 1995b). È questa la logica, il codice che guida la comunicazione politica e la distingue dagli altri ambiti della comunicazione, quali il diritto e la scienza. Se dunque il nostro discorso si colloca al livello del sistema scientifico (ma verrebbe da chiedersi: e dove altrimenti?) di una teoria sociologica del diritto, diventa possibile lavorare al nostro tema sia muovendoci nell'ambito dei criteri di scientificità del nostro approccio teorico, sia ragionando in termini di plausibilità giuridica delle questioni sollevate e delle possibili soluzioni, una plausibilità che include dunque anche i criteri di coerenza interna (quindi giuridici) del materiale normativo, compreso lo stesso giudizio di costituzionalità cui debbono soggiacere le decisioni del potere politico. Il paragrafo successivo illustrerà il nostro riferimento teorico in relazione alle dinamiche evolutive. Nel terzo, invece, vedremo come entra nelle nostre dinamiche la questione della tecnologia. È il quarto paragrafo che avrà ad oggetto la discussione delle implicazioni giuridiche dell'intelligenza artificiale. Nell'ultimo presenteremo le nostre conclusioni.

\section{EVOLUZIONE}

Il binomio diritto-società, che abbiamo richiamato precedentemente ad indicare la dinamica coinvolta dal nostro tema, deve essere precisata in merito al nostro obiettivo, 
tenuto conto del riferimento teorico costituito dalla teoria dei sistemi sociali e dalla sua comprensione della società moderna come società funzionalmente differenziata. ${ }^{5}$ Infatti, tale denominazione non deve trarre in inganno rispetto alla circostanza che il sistema giuridico è parte anch'esso della società e co-evolve insieme ad essa. Con ciò intendiamo chiarire, da un lato, che le dinamiche evolutive del sistema giuridico sono dinamiche che hanno il loro referente primario nei sistemi sociali e non in un presunto universo normativo alieno alla dimensione sociale. Non c'è dunque, da questo punto di vista, una contrapposizione tra normativo e fattuale, tra essere e dover essere, tra norme e fatti. ${ }^{6}$ Dall'altro lato, che in linea di principio, la sfera extra-sociale, non ha, e questo ha conseguenze sul nostro tema, effetti diretti sulla società e dunque neanche sul sistema giuridico, se non in senso distruttivo. Né si può parlare di una relazione diretta del tipo stimolo-risposta neanche nel caso delle connessioni tra il diritto e le altre sfere sociali. Piuttosto, dobbiamo immaginare, ricollegandoci al paragrafo precedente, una logica più complessa che consiste nell'attivazione di dinamiche che sono proprie dei singoli sistemi e tra loro differenziate. Il sistema politico ricostruisce la società al suo interno a partire dal suo punto di vista che consiste nella costruzione del problema sociale rispetto al quale (programmazione di scopo), vengono prodotte proposte di soluzione (proposte di legge). La selezione di una di queste in base al codice maggioranza/ opposizione in sede di discussione nell'ambito del procedimento legislativo diventa decisione collettivamente vincolante grazie al medium del potere. Il sistema giuridico, da parte sua, grazie all'accoppiamento strutturale con la politica realizzato dalla costituzione si trova a dover operare a un livello di complessità ridotto rispetto alla politica, per questo i procedimenti che caratterizzano le organizzazioni del sistema giuridico (per esempio i tribunali) sono di tipo differente. L'intervento politico infatti qui funge da "premessa decisionale" per il diritto, selezionando tra quelli possibili il contenuto che diventa norma. A partire da qui, il sistema giuridico dovrà decidere se e dove ricorrono le condizioni per l'applicazione della norma e dunque delle conseguenze previste. D'altra parte, questo rapporto tra politica e diritto non va inteso in senso gerarchico, né nel senso di una preordinazione della politica rispetto al diritto. Il rapporto, invece, è di tipo circolare: potremmo descrivere l'intero passaggio alla modernità dal punto di vista della trasformazione di rapporti lineari (verticalità: gerarchie) in circolarità. In questo caso si tratta della sostituzione della catena della legittimità (conosciuta anche come concezione discendente del potere) con un rapporto circolare realizzato dalla costituzione e in base al quale la politica pone il diritto, ma il diritto esercita il giudizio di legittimità costituzionale sulla decisione politica. In questo modo, ricollegandoci alla discussione in apertura di paragrafo, si realizza la separazione tra i due ambiti, ma allo stesso tempo anche il loro stretto legame. Una volta chiarito il rapporto tra politica e diritto, se noi ci muoviamo, come abbiamo anticipato, nell'ambito del sistema scientifico dovremo tener conto innanzitutto di un altro codice e altri programmi per la tecnicizzazione del codice e cioè altri media simbolicamente generalizzati, ${ }^{7}$ poiché quelli della politica e del diritto non sono qui rilevanti. Ma il sistema scientifico entra qui in gioco a più livelli come abbiamo 
visto. Se ci ricolleghiamo ad Hans Kelsen, che abbiamo più volte richiamato, vediamo come parallelamente alla definizione di quell'universo normativo (dover essere) specificamente rappresentato dal diritto rispetto all'universo fattuale (essere), la sua proposta teorica definisce la scienza giuridica come una scienza che possiede anch'essa i caratteri di normatività del suo oggetto, a differenza di una scienza come la sociologia che si occupa della descrizione della realtà, di ciò che accade (e di come mai accade ciò che accade) invece di ciò che deve accadere (KELSEN, 1967).

Tanto per dire che anche il nostro discorso potrebbe svolgersi al livello di quella particolare scienza che è la scienza giuridica, come una teoria della riflessione del sistema giuridico, per usare il lessico luhmanniano. Ma il punto a partire dal quale ci muoviamo non è quello della teoria del diritto, di una autosservazione del sistema giuridico ma di una eterosservazione, di una osservazione esterna, una osservazione sociologica del sistema giuridico, in questo caso delle trasformazioni di questo sistema innescate da uno sviluppo tecnologico. ${ }^{8}$ Della sua evoluzione, dunque, poiché è questo che intendiamo per evoluzione: la trasformazione delle strutture. Come abbiamo anticipato, in quanto sono da escludere rapporti puntuali con l'ambiente, sia con l'ambiente interno alla società, sia a maggior ragione con l'ambiente esterno, o relazioni causali, si tratta di osservare come il diritto segue su base autoreferenziale, per autocontatto quindi, l'evoluzione degli altri sistemi sociali, del suo ambiente sociale, rispetto al quale appare in coevoluzione. Sarà dunque rilevante osservare come sia possibile l'evoluzione di un singolo sistema e anche per quanto riguarda la tecnica dovrebbe essere utile definire in che senso essa abbia effetti sulla evoluzione della società. Nel prossimo paragrafo accenneremo all'importanza dell'evoluzione dei mezzi di comunicazione sulla forma prevalente della differenziazione sociale. D'altra parte, qui possiamo solo accennare al fatto che è difficile, se non impossibile determinare sviluppi singoli, pur dovendo ammettere un'assenza di coordinamento e di pianificazione per quanto riguarda la creazione delle tecnologie della comunicazione e le loro conseguenze sul successivo sviluppo sociale. Allo stato attuale non possiamo prescindere dal riconoscere che gli sviluppi tecnologici più recenti hanno ulteriormente avvicinato termini una volta contrapposti al concetto di tecnica: non solo la natura e la tecnica oggi tendono a confondersi ma la stessa umanità, una volta pensata come minacciata dal potere della tecnica, oggi può immaginare grazie ad essa la sua emancipazione dai propri limiti. Nella conclusione vedremo in che senso e con quali implicazioni per la società. Per ora basti anticipare che non possiamo più pensare alla tecnologia come a una mera applicazione della scienza (LUHMANN, 1998, p. 517-522).

\section{TECNOLOGIA}

Ci troviamo qui in un ambito particolare di articolazione della nostra società. Ciò che ci interessa è lo sviluppo tecnologico e l'evoluzione delle strutture comunicative che questo sviluppo innesca. Parlare di tecnica e tecnologia, significa, dal nostro punto di vista, prendere 
in considerazione una implicazione sociale di queste in quanto le rendiamo oggetto della comunicazione, tema della comunicazione. Tuttavia, la tecnologia ha una rilevanza di altro tipo rispetto alla comunicazione quando ci riferiamo alle tecnologie della comunicazione, ma anche per quanto concerne i nuovi sviluppi tecnologici. Questo ha ricadute notevoli sul nostro tema, ma andiamo con ordine.

Una descrizione dell'evoluzione della società moderna può essere effettuata osservando lo sviluppo dei media della comunicazione, anche se spesso i sociologi hanno trascurato questo aspetto, se si pensa che, afferma Luhmann (2015, p. 98-99), "le questioni legate alla nascita della scrittura sono state lasciate agli storici dell'antichità; se ne sono occupati anche gli etnologi e i linguisti." A partire dalla stampa del libro, ma secondo alcuni già con la scrittura, la tecnica ha messo a disposizione della società sempre nuovi e più efficaci "media della diffusione" che hanno consentito la riproduzione appunto tecnica della comunicazione, risolvendo specifici problemi. Il diritto e la politica, da parte loro, hanno "reagito" a questo sviluppo attraverso proposte di regolazione degli strumenti che si sono affermati a questo scopo. Istituti quali il copyright e l'authorship, i brevetti ecc., per esempio, con il tempo si sono dovuti adattare a nuove configurazioni rese possibili dall'uso del computer e di Internet. Si tratta di sviluppi che, da un altro punto di vista, hanno dato vita all'idea che si tratti di tecnologie, "di sviluppi tecnologici" dice Luhmann (2015, p. 100): "e che tutto il resto sarebbe una loro conseguenza collaterale"; e d'altra parte, conclude, come nel caso della tecnica, neanche il concetto di tecnologia è stato chiaramente definito.

Dal punto di vista che ci interessa, la teoria dei sistemi offre un potente strumento utile a collocare la tecnologia e gli sviluppi tecnologici in quadro teorico più ampio e coerente rispetto al problema che stiamo trattando. Ci muoviamo, lo ripetiamo, nell'ambito del sistema sociale complessivo della comunicazione, caratterizzato dalla differenziazione funzionale di sottosistemi quali la scienza, il diritto, la politica, l'educazione, l'economia, la religione, per citare solo i maggiori, tra i quali non esistono rapporti gerarchici. Quando parliamo di evoluzione del diritto, abbiamo a che fare con una differenza tra sistema e ambiente, che in questo caso è riconducibile alla differenza tra il sistema del diritto e il suo ambiente costituito da tutti gli altri sottosistemi sociali, mentre l'ambiente esterno alla società è rimasto finora sullo sfondo. Tuttavia, una definizione della tecnologia che mutuiamo da Luhmann (1998), rimette in gioco anche l'ambiente esterno nelle dinamiche che stiamo osservando. Infatti, è possibile, dal punto di vista sociale, definire la tecnologia come un ambito della comunicazione che ha la funzione di intervenire sull'ambiente fisico, chimico e biologico. ${ }^{10}$

Questa definizione ci consente di introdurre quella innovazione specifica costituita dalla intelligenza artificiale. Ci stiamo spostando dalle ricadute dell'innovazione tecnologica sullo sviluppo dei media della comunicazione ad un nuovo paradigma tecnologico con implicazioni di natura ben differente. Già il computer e Internet definiscono chiaramente un ambito di sviluppo che consiste nell'incremento della capacità (limitata) umana di elaborazione della informazioni (RHEINGOLG, 1985; CASTELLS, 1996, 2001). Con Herbert Simon avevamo 
assistito ad una svolta nello studio del comportamento decisionale nelle organizzazioni, che consiste in una critica dell'approccio normativo della economia classica e nello sviluppo di un approccio descrittivo al processo decisionale che mette appunto in discussione il decisore olimpico dotato di una razionalità assoluta ipotizzato dalle teorie classiche (SIMON, 1985). Simon, infatti, non solo ha ridefinito l'ambiente della scelta, ma anche il soggetto della scelta e questo proprio sulla base di una osservazione del comportamento decisionale concreto all'interno dei contesti decisionali delle organizzazioni. Il soggetto umano, allora, per Simon, all'ora della scelta, appare dotato, piuttosto, di una razionalità che risulta limitata in virtù della sua incapacità, dal punto di vista cognitivo, di elaborare tutte le informazioni necessarie a massimizzare i propri obiettivi (SIMON, 1967).

Se dunque il computer e Internet hanno costituito un fattore decisivo verso l'incremento della capacità umana di elaborazione delle informazioni, negli ultimi anni si è assistito a sviluppi straordinari in questa direzione. Ci riferiamo alle così dette tecnologie convergenti o tecnologie NBIC (nanotechnology, biotechnology, information technology and cognitive science), una convergenza tecnologica tra ambiti di ricerca una volta distinti e che ha ricadute sorprendenti in termini di human enhancement nella medicina, per esempio, cioè della possibilità di mettere in atto interventi tesi al "potenziamento umano". ${ }^{11}$

Da qui, limitatamente al nostro tema, due questioni ci appaiono rilevanti: da un lato, le conseguenze di questa convergenza sulla evoluzione dell'ambiente della società, che riprenderemo nelle conclusioni; dall'altro lato, un caso specifico di questo sviluppo tecnologico rappresentato dall'intelligenza artificiale e dalle problematiche che pone al diritto in relazione alla sua regolamentazione giuridica, che svilupperemo nel paragrafo successivo.

Con i termini "intelligenza artificiale" ci si riferisce tanto a una disciplina di studio, in questo caso appartenente all'informatica, quanto all'oggetto studiato. Potremmo definire i sistemi hardware/software dotati di Intelligenza Artificiale, come dei sistemi in grado di realizzare prestazioni che, a un osservatore comune, sembrerebbero essere di pertinenza esclusiva dell'intelligenza umana (SOMALVICO et al., 2003). Tale parvenza di umanità rinvenibile nelle prestazioni di sistemi dotati di IA, può essere raggiunta tramite diversi paradigmi tecnologici. Il machine learning, ad esempio, permette a un computer di apprendere da dati presenti in rete modalità di risoluzione di problemi nuove rispetto a quelle individuate in fase di programmazione. Senza addentrarci oltre in tali definizioni per ragioni di spazio, proprio l'ultimo punto risulta pregno di interesse per noi che scriviamo ma anche per i sistemi sociali già citati, il diritto e la politica: quali sono le implicazioni della nascita di sistemi hardware/ software in grado di rendere possibili da sé nuove operazioni non contemplate dallo sviluppatore/progettista?

\section{INTELLIGENZA ARTIFICIALE}

Potremmo essere alle soglie di una svolta epocale tale da mettere in discussione gli 
strumenti finora utilizzati per analizzare la società e i suoi sistemi sociali. Lo sviluppo tecnologico, che fino a ieri ha fornito moltissimi strumenti agli esseri umani tali da facilitare la vita e rendere possibile operazioni prima impensabili, starebbe per dare la vita a "oggetti" dotati di una qualche forma di pensiero autonomo. Questa è una dichiarazione forte e non condivisa da tutti. Vi è infatti chi sostiene che l'intelligenza artificiale non possa mai spingersi oltre rispetto alla risoluzione, neppure brillante, di piccoli problemi senza mai arrivare a sviluppare un'autocoscienza (SEARLE, 1997). Non potendo affrontare in così poche righe una questione di tale complessità, ci si può limitare a porre un dubbio a coloro che affermano di avere così granitiche certezze: non è forse in tale modo, provando cioè a risolvere specifici problemi (ad esempio, reperire cibo, sfuggire ai predatori [...]), che si sono sviluppate magnifiche forme di vita sul nostro planeta fino ad arrivare allo stesso essere umano?

Faremo dunque largo uso del condizionale poiché l'ambito in cui ci muoviamo non è ancora chiaramente definito e le certezze sono veramente poche.

Tuttavia non possiamo esimerci dal constatare che auto a guida autonoma (TESLA, 2017), software che scrivono brani musicali (TENSORFLOW, 2017), robot che svolgono compiti di assistenza agli anziani (ROBOT-ERA, 2017) sono oggi evoluzioni tecnologiche già acquisite.

Dal punto di vista dei sistemi sociali, esse costituiscono una evoluzione del loro ambiente e ne dovranno tener conto. Un primo passo in tale direzione ci pare sia stato fatto con l'approvazione della Risoluzione del Parlamento europeo del 16 febbraio 2017 recante raccomandazioni alla Commissione concernenti norme di diritto civile sulla robotica (2015/2103 (INL)). In tale documento si asserisce che

[...] nell'ipotesi in cui un robot possa prendere decisioni autonome, le norme tradizionali non sono sufficienti per attivare la responsabilità per i danni causati da un robot, in quanto non consentirebbero di determinare qual è il soggetto cui incombe la responsabilità del risarcimento né di esigere da tale soggetto la riparazione dei danni causati (EUROPEAN PARLIAMENT, 2017, online).

Inoltre si arriva a contemplare l'ipotesi di istituire

[...] uno status giuridico specifico per i robot nel lungo termine, di modo che almeno i robot autonomi più sofisticati possano essere considerati come persone elettroniche responsabili di risarcire qualsiasi danno da loro causato, nonché eventualmente il riconoscimento della personalità elettronica dei robot che prendono decisioni autonome o che interagiscono in modo indipendente con terzi (EUROPEAN PARLIAMENT, 2017, online).

I passi appena riportati mettono in luce l'inadeguatezza dell'attuale sistema normativo rispetto a tali innovazioni, ma, d'altra parte, costituiscono tentativi di abbozzo di nuove norme che affrontino questi cambiamenti.

Varie sono le possibili nuove norme giuridiche che il documento citato a inizio paragrafo propone come soluzioni. La più affascinante e pregna di conseguenze è proprio l'attribuzione di una qualche forma di soggettività a questi nuovi "esseri". Tuttavia, tale soluzione potre- 
bbe sollevare nuove questioni che coinvolgono concetti e istituti posti alle fondamenta del sistema giuridico e frutto di evoluzioni millenarie: tramonterà nuovamente l'idea di soggetto di diritto unico in favore della presenza nel sistema giuridico di una pluralità di soggetti individuati da status differenziati sulla base di criteri diversi rispetto a quelli delle società pre-moderne: ma quali?

Assumendo il punto di vista della teoria dei sistemi, si potrebbe quasi affermare la neutralità del diritto rispetto all'eventuale nascita di esseri senzienti diversi da ciò che biologicamente si può individuare come essere umano. Infatti la teoria luhmanniana, espellendo i sistemi psichici dall'analisi del diritto come sistema sociale, si è resa immune a tali mutazioni. In altre parole che una sentenza sia resa da un giudice dotato di toga e martelletto o da un software dotato di intelligenza artificiale risulterebbe del tutto indifferente. Si tratterebbe pur sempre di operazioni giuridiche rese possibili dal sistema del diritto e di cui questo sarebbe pur sempre il risultato.

\section{CONCLUSIONI}

Il caso dell'intelligenza artificiale proposto nell'ultimo paragrafo del presente articolo induce ad una riflessione circa lo iato temporale tra evoluzioni tecnologiche e operazioni giuridiche. Il documento approvato dal parlamento europeo infatti non è affatto una norma giuridica, quanto un atto politico di impulso rivolto a un organo politico, la Commissione Europea, al fine di formulare una norma giuridica che, ad oggi ancora non esiste. Il gap temporale è frutto da un lato del rapporto, neppure stretto, tra diritto e politica, dall'altro dello stesso funzionamento della politica. Il rapporto diritto-politica, abbiamo visto, è circolare e ciò comporta che, nel momento in cui il diritto "ravvisa" la necessità di nuove norme deve attendere la politica oppure risolvere da sé i conflitti che sorgono e i casi che si presentato in qualche modo, ma: come ${ }^{12}$ Le procedure, sappiamo, necessitano tempo per formarsi e arrivare a una conclusione, che potrebbe anche non giungere, ma anche questo fa parte del funzionamento democratico. Ciò porta a una differenza temporale tra il momento in cui una nuova tecnologia vede la luce e il momento in cui si avrà una norma che vi si possa applicare. Ma la norma in questione, al momento della sua emanazione non sarà certamente riferita alla tecnologia esistente in quel momento, giacché nel frattempo tale tecnologia si sarà ulteriormente evoluta. Sembra che le norme dell'oggi siano destinate a regolare la tecnologia di ieri (SARACENO, 2015). D'altra parte, ci chiediamo se si debba sempre intervenire politicamente su ogni nuova tecnologia; dati gli ultimi sviluppi, in realtà, questo sembrerebbe non solo utile, ma necessario.

Nonostante le trasformazioni strutturali e l'evoluzione semantica siano tra loro correlate, anche qui assistiamo ad un "ritardo": il patrimonio concettuale idoneo a descrivere la società si stabilizza solo a distanza di tempo rispetto alla struttura sociale che pretende di descrivere. Sembra un fatto inevitabile. Una concettualità, per acqui- 
sire plausibilità e accettazione sociale diffusa deve necessariamente "attendere" che le novità, le deviazioni dalla norma, si siano in qualche modo stabilizzate (BARALDI; CORSI; ESPOSITO, 1987).

E in ogni caso, per ciò che riguarda la semantica, ci muoviamo sempre in ambito sociale, nell'ambito della comunicazione, delle sue strutture e dei suoi media. Con la tecnologia, viceversa dobbiamo mettere in conto aspetti ulteriori. Fra questi il fatto che con la convergenza NBIC i confini tra scienze della vita e scienze hard, tra biologia e macchine convergono, come mostra il caso della intelligenza artificiale dove una macchina acquisisce caratteristiche proprie dell'intelligenza umana. A ciò va aggiunto il fatto che in questo modo, con riferimento al human enhancement, per esempio, la medicina sembra chiamata ad eccedere la sua funzione, proprio in quanto non deve occuparsi solo della cura delle malattie (MARIN, 2012; CORSI, 2015).

Qualcosa del genere si può notare anche per le prove scientifiche ammesse all'interno del processo penale. Si tratta di una pratica sempre più diffusa che ha finito per interessare anche metodologie non ancora comprovate all'interno della comunità scientifica, di fatto, in questo modo, "scaricando" sul diritto l'onere di attestarne la scientificità, cosa che evidentemente non appartiene ai suoi compiti.

Se trasformiamo dal nostro punto di osservazione le preoccupazioni per il "governo" delle scoperte scientifiche e del progresso tecnologico, non solo quelle di tipo etico, ma anche quelle relative alla capacità della società di seguirne le altre conseguenze a livello sociale, allora vediamo che il problema si pone nei termini, affatto tranquillizzanti, che possono essere espressi dalla domanda: la società complessivamente considerata sarà in grado di "sopportare" la rapidità dei cambiamenti innescati dal processo tecnologico?

In altri termini, se contestualizziamo il discorso nell'ambito dei processi evolutivi, possiamo vedere come, sulla scorta di una preoccupazione, non solo teorica, posta da Luhmann, la questione diventa quella di sviluppi parziali, non coordinati con la evoluzione della società, ma quasi indipendenti da essa e della capacità strutturale della società di accoglierli nella forma attuale, di cambiarla in conseguenza di questi (ma come?) o di non sopravvivere al loro impatto. Qui dobbiamo aggiungere che l'ambito particolare della intelligenza artificiale introduce una suggestione particolare al tema generale. Difatti qui possiamo assistere alla rarefazione stessa, potremmo dire, dei confini tra le scienze e tra i loro oggetti, tra scienza pura e applicata, tra science and humanities, tra la biologia, abbiamo detto, e la tecnologia, ma anche tra le macchine e gli esseri umani. ${ }^{13}$ Ciò in qualche modo sembra poter prefigurare addirittura una sorta di co-evoluzione tra il potenziale umano e quelle che abbiamo indicato come tecnologie convergenti, attraverso l' "innesto" di nuovi strumenti nell'architettura umana. ${ }^{14}$

E il diritto, come si inserisce in questo quadro dove ben altre emergenze sembrano richiamare l'attenzione? La situazione di partenza che ha caratterizzato il nostro discorso sembra qui non essere più la stessa. Avevamo preso le mosse dal fatto che fosse possibile indicare caratteristiche specifiche per ciascuno degli ambiti di articolazione della società, 
anche per il diritto, la scienza e la tecnologia e ciò, tuttavia, sempre a partire da una unità pregressa rispetto alla quale tracciare differenze. Questa unità per i sistemi sociali sappiamo essere la comunicazione. Questa unità è al tempo stesso ciò che differenzia la società rispetto a tutto il resto, ad altre strutture della realtà, a ciò che chiamiamo ambiente della società, a ciò che non è comunicazione. Anche in base a questo abbiamo preso in considerazione una evoluzione della società che non ha referenze con l'evoluzione dell'ambiente. L'ambiente evolve più lentamente della società. Ora, però, alla fine del nostro discorso, ci troviamo con un quadro della situazione che sembra corrodere questi presupposti, la stessa differenza tra sistema della società e suo ambiente.

All'inizio del nostro saggio ci eravamo posti questioni quali: come evolve la società? il cambiamento tecnologico produce evoluzione sociale? è possibile una evoluzione non coordinata tra i sistemi sociali? Ora da un punto di vista giuridico, i quesiti connessi alle nuove acquisizioni tecnologiche spingono ad un approccio de jure condendo, sollevando altri interrogativi: a chi si potrebbe attribuire la paternità di un'opera intellettuale creata da un software dotato di intelligenza artificiale? E di conseguenza: a chi si potranno attribuire i diritti di sfruttamento economico di tale opera? a chi verranno imputati i danni creati dalle macchine nell'esercizio del proprio "libero arbitrio"? chi avrà il diritto di ricevere gli stipendi maturati dal lavoro svolto da tali nuovi "lavoratori"? E per concludere, con le parole di Luhmann, "Se siamo in grado di produrre esseri viventi mediante la manipolazione genetica, possiamo poi anche brevettarli?" (LUHMANN, 2015, p. 194).

\title{
Soundtrack: Arvo Pärt, Da Pacem
}

\section{LAW, SCIENCE AND TECHNOLOGY}

\begin{abstract}
The new information and communication technologies play a relevant role for the social systems involved. For this reason, the theoretical description of society cannot neglect the growing dependency on technology by the information society. Our article is aimed at examining technological progress through the point of view of the evolution of legal instruments that regulate new technologies. It embodies a legal and sociological point of view in which the dynamics of the evolution of Law regarding the evolution of society is at play. Unlike other sectors of Law, the classic example being Family Law, here there is no consolidated tradition to which one can report or which one can reject. This means that the solutions must be "invented" in light of the new paradigms that, appealing to fundamental legal institutes, have a visionary capacity, that are, as Luhmann says, "pregnant with the future". Our discourse will thus stem from a general theoretical placement of the dynamics
\end{abstract}


among Law, technology and society to conclude having as reference the specific case of artificial intelligence.

Keywords: Technological progress. Social evolution. Law and Society. Human enhancement technology. Artificial intelligence.

\section{DERECHO, CIENCIA Y TECNOLOGÍA}

\section{RESUMEN}

Las nuevas tecnologías de información y comunicación tienen un rol relevante para los sistemas sociales involucrados. Por esa razón, la descripción teórica de la sociedad no puede descuidar a la creciente dependencia de la sociedad de la información en la tecnología. Nuestro artículo se propone a examinar el progreso tecnológico desde el punto de vista de la evolución de instrumentos jurídicos que regulan nuevas tecnologías. Él personifica un punto de vista jurídico y sociológico en que se analiza la dinámica de la evolución del Derecho en relación a la evolución de la sociedad. A diferencia de otros sectores del Derecho, el ejemplo clásico es el Derecho de Familia, acá no hay una tradición consolidada a la cual se puede recurrir o rechazar. Eso significa que las soluciones deben ser "inventadas" a la luz de los nuevos paradigmas que, haciendo apelo a institutos jurídicos fundamentales, tienen capacidad visionaria, que están, como lo dice Luhmann, "grávidos del futuro". Nuestro discurso partirá entonces de un encuadramiento teórico general de la dinámica entre el Derecho, la tecnología y la sociedad para concluir teniendo por referencia el caso específico de la inteligencia artificial.

Palabras clave: Progreso tecnológico. Evolución social. Derecho y sociedad. Tecnología de mejoría humana. Inteligencia artificial.

1 "Parte del presente lavoro è stata sviluppata durante un soggiorno di ricerca presso il Centro de Estudios Politicos y Constitucionales (CEPC) di Madrid, realizzata tra ottobre 2017 e gennaio 2018”. (Nota do Editor: A Revista Opinião Jurídica agradece ao Sr. José Ilo Mota, responsável pela revisão da língua italiana.)

2 La norma fondamentale, sappiamo, non ha i caratteri della giuridicità, non essendo una norma scritta, ma presupposta e quindi non facente parte dell'ordinamento giuridico. Cfr. (KELSEN, 1967).

3 Cfr., tra gli altri, (DE GIORGI, 1998; CORSI, 2001).

4 Già qui dovremmo citare anche le scienze cognitive, ma non sapremmo dove collocarle. D'altra parte, come vedremo più avanti, si tratta proprio di questo: della rottura di paradigmi consolidati, di differenze stabilizzate, di nuovi orizzonti che non si lasciano ricondurre a schematismi noti.

5 Per il concetto di differenziazione funzionale, si veda (LUHMANN; DE GIORGI, 1994).

6 Cfr. a questo proposito, la differenza tra fattuale e normativo in Hans Kelsen: op. cit (KELSEN, 1967).

7 Ci riferiamo qui ad un concetto centrale delle teoria dei sistemi: cfr. (LUHMANN, 2015, p. 124-152). 
8 Questo approccio è ben diverso da quello della scienza giuridica, come abbiamo cercato di chiarire: cfr., a questo proposito. (LUHMANN, 2002, p. 37-45).

9 Cfr. (THOMPSON, 1998; LUHMANN, 2015). A proposito della tecnica, Luhmann fa notare come "i processi vitali sulla terra dipendono sempre più dalla tecnica e l'approvvigionamento di una parte sempre maggiore della popolazione dipende da possibilità produttive incrementate dalla tecnica", d'altra parte, continua, non c’è una rappresentazione adeguata di tutto questo, "perché la tecnica non è semplicemente una trasposizione della scienza, ma si sviluppa con una dinamica propria a seconda di dove un bisogno e un mercato di prodotti tecnici sia riconoscibile" (LUHMANN, 2015, p. 62).

$10 \mathrm{Su}$ questo punto si intrecciano ambiti tra loro apparentemente lontani della comunicazione, come anche concetti distinti della teoria. Infatti, trattando dell'influsso dei media sull'evoluzione, Luhmann associa la tecnologia all'educazione e alla medicina rispetto alla loro funzione di produrre effetti sull'ambiente umano (LUHMANN, 1998, p. 407)

11 Cfr., fra gli altri, (GUARINO, 2015; MARIN, 2012).

12 A questo proposito alcuni sottolineano il ruolo fondamentale della giurisprudenza nel contesto, per esempio, delle tecnologie NBIC, proprio per la rapidità del progresso tecnologico, che rende quasi immediatamente obsoleta ogni norma in materia: cfr. (GUARINO, 2015).

13 Si è parlato a questo proposito di post-umano e di post-umanesimo; cfr. per esempio (GUARINO, 2015).

14 Cfr. (ROCO, 2004); nello stesso volume cfr. anche (KHUSHF, 2004).

\section{REFERÊNCIAS}

BARALDI, Claudio; CORSI, Giancarlo; ESPOSITO, Elena. Semantica e comunicazione. Bologna: Clueb, 1987.

BERNSTEIN, Jeremy. Uomini e macchine intelligenti. Milano: Adelphi, 1990.

CASTELLS, Manuel. The Information Age I. The Rise of Network Society. Oxford: Blackwell, 1996.

. The Internet Galaxy. Oxford: Oxford University Press, 2001.

CORSI, Giancarlo (a cura di). Salute e malattia nella teoria dei sistemi. Milano: Franco Angeli, 2015.

Sociologia da Constituição. Revista da Facultade de Direito da Universidade Federal de Minas Gerais, Minas Gerais, n. 39, p. 169-189, 2001.

DE GIORGI, Raffaele. Scienza del diritto e legittimazione. Lecce: Pensa MultiMedia, 1998.

ESPOSITO, Elena. L'operazione di osservazione. Milano: Franco Angeli, 1993.

EUROPEAN PARLIAMENT. Risoluzione del Parlamento europeo del 16 febbraio 2017 recante raccomandazioni alla Commissione concernenti norme di diritto civile sulla robotica (2015/2103(INL)). Europarl, Strasburgo, 16 febbr. 2017. Available in: <http:// www.europarl.europa.eu/sides/getDoc.do?pubRef=-//EP//TEXT+TA+P8-TA-2017$0051+0+\mathrm{DOC}+\mathrm{XML}+\mathrm{V0} / / \mathrm{IT}>$. 
GUARINO, Carmelo. Tecnologie NBIC, medicina, diritto. Sociologia del diritto, n. 2, p. 93-114, 2015.

KELSEN, Hans. Lineamenti di dottrina pura del diritto. Torino: Einaudi, 1967.

KHUSHF, George. System Theory and the Ethic of Human Enhancement: A Framework for NBIC Convergence. Annals of the New York Academy of Sciences, v. 1013, p. 124149, maggio, 2004.

LUHMANN, Niklas; DE GIORGI, Raffaele. Teoria della società. Milano: Franco Angeli, 1994.

LUHMANN, Niklas. Osservazioni sul moderno. Roma: Armando, 1995a. . Procedimenti giuridici e legittimazione sociale. Milano: Giuffrè, 1995b. . La costituzione come acquisizione evolutiva. In: ZAGREBELSKY, Gustavo; PORTINARO, Pier Paolo; LUTHER, Jörg. Il futuro della costituzione. Torino: Einaudi, 1996. p. 83-127.

. Das Recht der Gesellschaft. Francoforte: Suhrkamp, 1997.

. Die Gesellschaft der Gesellschaft. Francoforte: Suhrkamp,1998.

. I diritti fondamentali come istituzione. Bari: Dedalo, 2002.

. Introduzione alla teoria della società. Lecce: Pensa Multimedia, 2015.

MARIN, Francesca. Il bene del paziente e le sue metamorfosi nell'etica biomedica. Milano: Bruno Mondadori, 2012.

RHEINGOLD, Howard. Tools for Thought: the history and future of mind-expanding technology. New York: Simon and Schuster, 1985.

ROBOT-ERA. Robot-Era. 2017. Disponibile in: 〈http://www.robot-era.eu/>.

ROCO, Michail C. Science and Technology Integration for Increased Human Potential and Societal Outcomes. Annals of the New York Academy of Sciences, v. 1013, p. 1-16. magg. 2004.

SARACENO, Chiara. Quando le leggi vanno piano accelerano i magistrati. La Repubblica, 20 genn. 2015.

SEARLE, John R. The Mystery of Consciousness. New York: New York Review of Books, 1997.

SIMON, Herbert A. Il comportamento amministrativo. Bologna: il Mulino, 1967. . Causalità, razionalità, organizzazione. Bologna: il Mulino, 1985.

SOMALVICO, Marco et al. Intelligenza Artificiale. Storia della scienza, Roma, v. 9, p. 615-624, 2003. 
TARELLO, Giovanni. Storia delle cultura giuridica moderna. Assolutismo e codificazione del diritto. Bologna: il Mulino, 1976.

TENSORFLOW. Machines. 2017. Disponibile in: <https://www.tensorflow.org/maanchehttp://www.flow-machines.com/>.

TESLA. Pilota automatico. 2017. Disponibile in: 〈https://www.tesla.com/it_IT/autopilot〉. THOMPSON, John B. Mezzi di comunicazione e modernità. Bologna: il Mulino, 1998. VON FOERSTER, Heinz. Sistemi che osservano. Roma: Astrolabio, 1987.

ZAGREBELSKY, Gustavo; PORTINARO, Pier Paolo; LUTHER Jörg. Il futuro della costituzione. Torino: Einaudi, 1996. 